\title{
Implementation of a Strengths-Based Approach to Teaching in an Elementary School
}

\author{
Keith Brownlee \\ Lakehead University \\ Edward P. Rawana \\ Lakehead University \\ Julia MacArthur \\ Lakehead University
}

\begin{abstract}
Schools play a significant role in addressing children's mental health needs and this article contends that schools can further contribute to student mental well-being by adopting a strengths perspective model. A specific strengths assessment and treatment model is presented that extends to individual, peer and group interventions as well as discussions within the classroom that is applicable to every student in the school and not only students considered "at risk." By engaging an entire class, or indeed an entire school, in a dialogue of strengths, the concept of strengths can become a part of the culture of the school and lead to a positive school environment. This article provides an overview of the model, its implementation in a school, including the theory informing the interventions, followed by two brief case studies showing how the model was applied in a classroom. The intervention not only transforms the way in which educators interact with students, but it changes the way students perceive themselves and the manner in which they perceive their own potential.
\end{abstract}

When working with children and youth, social workers and educators are on the front line together, but often have different concerns and different methods of addressing those concerns. One goal that unifies the two professions is the desire for children and youth to fulfill their potential and achieve their dreams. Unfortunately, these positive intentions are often overshadowed by orientations to helping that emphasize the shortcomings, deficits, and pathology of children. The strengths perspective within social work (Glicken, 2004; Saleebey, 1997, 2008), however, eschews the deficit-based traditions of medical model orientations and has emerged as a credible framework for aiding children and their families in addressing life challenges (Tedeschi \& Kilmer, 2005). This paper presents a specific strengths perspective model that can be integrated into schools as a viable method to enhance the support that students receive at school and complementing a pathology based framework. 
The strengths perspective can offer many advantages to educators that extends the resilience paradigm currently accepted within educational systems (Masten, Herbers, Cutuli, \& Lafavor, 2008; Stewart, Sun, Patterson, Lemerle, \& Hardie, 2004). The strengths perspective promotes the community acknowledgement and self-recognition of children's personal assets and encourages them to use these assets to achieve their goals, overcome their problems, and contribute to their community. It is an approach that has not been well established in educational settings, except in the context of school counselling and school psychology (Saleebey, 2008; Jimerson, 2004) and in working with emotional and behavioral disorders (Farmer, Farmer, \& Brooks, 2010). However, the strengths perspective has recently been formulated into a methodical framework for use in assessment and treatment that has been successfully implemented in the classroom learning environment (Rawana et al., 2009).

This paper discusses the strengths, assessment, and treatment model described by Rawana and Brownlee (2009) as it has been extended and applied to an inner city elementary school. Differences between the strength-based approach to teaching and the resiliency education paradigm will be discussed, followed by a description of the stages of the strengths, assessment and treatment model, how it has been implemented by educators, and the resulting strengthsbased approach within the classroom. Two brief case studies are introduced to further elaborate and illustrate the influence of the strengths-based approach on the students and the school community and the potential this approach has for contributing to a positive teaching environment.

\section{Theoretical Foundation of Strengths and Resiliency}

There have always been people who have overcome adversity, surviving life's obstacles, such as, poverty or even thriving despite the hardships they faced (for example, Werner \& Smith, 1992). The research exploring factors associated with such success formed the foundation of resiliency theory (Howard, Dryden \& Johnson, 1999). Resiliency has been defined as "a dynamic process encompassing positive adaptation within the context of significant adversity" (Luthar, Cicchetti, \& Becker, 2000, p. 543). Resiliency is concerned with the surprisingly common processes involved in individuals overcoming significant life challenges (Masten, 2001) and uses the concepts of risks and protective factors to determine who is resilient and why. Masten outlines exclusion criteria for being considered resilient: "Individuals are not considered resilient if there has never been a significant threat to their development; there must be current or past hazards judged to have the potential to derail normative development. In other words, there must be demonstrable risk" (p. 228). However, resiliency is not "a single dichotomous variable" (Condly, 2006, p. 213). Most individuals do not simply fit the categories of resilient or not resilient; resiliency is a more fluid, relative, and continuous process. In addition, risks and protective factors themselves are complex, and what is a risk for one person could potentially be a protective factor or even a neutral experience for someone else (Ungar, Brown, Liebenberg, Cheung, \& Levine, 2008). As resiliency can only exist when there are demonstrable risks, the resiliency paradigm is narrowed to some extent by the need to draw on assets that are based on overcoming those risks. The strength perspective and the strength, assessment and treatment model, as operationalized 
by Rawana and Brownlee (2009), however, offer an alternative paradigm that draws on children's resources and assets whether or not these resources and assets emerged in relation to challenges or adversity within a child's life.

The strengths perspective is part of a much larger movement representing a departure from the "deficit model" of treatment that has been embraced under different names by many different professions, including health (wellness), child and youth work (youth development), special education (assets), early childhood education and psychology (child development), and substance abuse programming (protective factors) (Brown, D'Emidio-Caston, \& Benard, 2001). The strengths perspective is based on the idea that everyone has strengths and that everyone has the potential to use these strengths to achieve personal goals. The strengths perspective also includes the assumption that by using their strengths students can achieve a better quality of life, even if the strengths the students use do not represent conventional knowledge, capacities, and resources (Saleebey, 2006). In the strength, assessment, and treatment model, strengths are defined as "a set of developed competencies and characteristics that is valued both by the individual and society and is embedded in culture" (Rawana and Brownlee, 2009, p. 256). This definition, besides recognizing a wide array of strengths, acknowledges that not all adaptive or survival skills are considered strengths by a community.

Another benefit of using the strength, assessment, and treatment model is that it has the potential to be applied on any scale, from the individual to school-wide programs. When the broader, group interventions are undertaken by school communities, the school-wide initiatives can work toward encouraging a culture of strengths in a school (Brownlee, Rawana, MacArthur \& Probizanski, 2010). If school-wide initiatives are teamed with the application of strengths in the classroom, students, staff, and families can become immersed in the vocabulary and culture of strengths that become reinforced throughout the school community. This assists all stages of the strength, assessment, and treatment model because everyone in the community tends to "speak the same language" and potentially contributes to the interventions. The culture of strengths becomes self-referential, and strengths can be recognized and utilized by educators, principals, caregivers, support staff, and by peers.

The strength, assessment and treatment model develops from assessment to intervention by moving through the stages of engagement, exploration, expansion, and evolution (Rawana \& Brownlee, 2009). Each of these stages has a particular function in the realization and utilization of students' strengths. The process includes educators and students, and is not something that one "does" to someone else. Including both educators and students is one way that the strengths, assessment, and treatment model can differ from resiliency-based interventions in schools, such as, the Resilience Education program, which is based upon "the opportunity, if not the responsibility, to shift how [educators] perceive, act, interact, and react with young people" (Brown et al., 2001, p. 31). The strengths, assessment, and treatment model recognizes that it is not only educators who sometimes have the tendency to look at situations from a deficit perspective, but also principals, policies, families, and students themselves. Thus, the model encourages a shift in perspective in multiple contexts of students' lives. In fact, the students rather than the educator frequently direct the 
implementation. It is a process of discovery and appreciation of the intrinsic value and importance of all members of the classroom, and the school community.

The strengths, assessment, and treatment model that is presented in this paper was implemented by educators at an inner-city elementary school in northwestern Ontario. Consistent with the strength model the program was formulated on the premise that all students and their families have strengths and that those strengths emerge from any aspect of the students' day-to-day lives. The school implemented the model school-wide as well as within individual classrooms. An account of the school-wide initiatives can be found elsewhere (cf., Brownlee, et al., 2010). The next section presents an overview of the model and explains the implementation of the model and the theory behind the interventions, followed by two brief case studies that give examples of how the model was applied by one teacher, Cathy (fictitious name), in a specific classroom.

\section{Strengths-Based Approach in the Classroom}

\section{Engagement}

The first stage of implementing the strengths, treatment, and assessment model is the engagement stage, in which the educator works on creating a positively oriented relationship with students, and with caregivers and any other significant people in the students' lives (Rawana \& Brownlee, 2009). While teachers value engagement as a critical component of working with students, the strength approach provides an easy roadmap that allows the student to promote the things that are good about them. The emphasis in this model, thus, is on discovering and discussing the students' strengths. The model establishes positive expectations, and the educator's relationships with children and parents can centre on a child's positive attributes, rather than on any perceived or residual deficits that may have previously been encountered in relation to the school. This does not mean that ongoing issues cannot be addressed or are minimized. However, engaging students and families with a strength orientation first can deal with issues that are assessed during the strengths, assessment, and treatment intervention. Engagement is the process of setting the tone of subsequent relationships, which usually last throughout the school year and often beyond into subsequent years. It is a continual process, working towards "the ongoing understanding and appreciation that the [educator] is working with the child and family on their behalf from a position of their strengths" (Rawana \& Brownlee, 2009, p.257). Positive and effective engagement enables the classroom to be regarded as an affirming social context (Fredricks, Blumenfeld, \& Paris, 2004) and the school itself to be viewed positively (Osterman, 2000), which is more likely to be reinforced at home by caregivers who feel that their children are being positively recognized and appreciated. Positive engagement is also more likely to foster parental involvement with the school, which has been associated with academic benefits for children, especially children from economically disadvantaged families (Cooper \& Crosnoe, 2007).

Once the strengths intervention has been established throughout the school, all students will have received a strengths assessment. The strengths assessment results provide a springboard of strengths from which to launch a year-long 
relationship with students. The strengths assessment results will also be able to be used by a new teacher when students move to the next grade. This process was noted by Cathy, as follows: "I would love to, at the beginning of the year, sit down and see, this is my new class; this is what they think their strengths are, and build on that. It's also a good way to get to know kids, and to have them come in on such a good note."

A further important application of the ongoing engagement stage is in its use with the significant people in students' lives. The principles behind engagement can effectively be used with caregivers, which is significant because under normal circumstances educators may not have frequent interactions with parents. In the regular times each year when contact is made, strength-based engagement is an effective way to create and maintain the positive relations and open communication that benefits the students, and to minimize possibilities of communication breakdown (Rawana \& Brownlee, 2009). With caregivers aligned with their students' needs and educators positively aligned with both students and caregivers, a strong support system can be built that can withstand the challenges faced by students as well as provide more resources for students across contexts, with home life and school life working in tandem.

\section{Exploration}

With students engaged in an ongoing strengths-based relationship with educators, the next stage of the strengths intervention is exploration. This stage is when the formal and comprehensive assessment of a student's strengths occurs. A strengths assessment can be completed by multiple people in the student's life, spanning multiple contexts of the student's life, such as, school, home, and recreation (Rawana \& Brownlee, 2009). It is not conventional for schools to compile non-academic assessments of students unless a school psychologist does it; however, the benefits of the assessment warrant the use of a formal strengths questionnaire. The assessment protocol is straightforward and because the student can complete the questionnaire, it can be done in the classroom. In addition to the central message to the student that the educators are interested in their strengths both inside and outside of school, a significant benefit to the educator is that the assessment contains information to which educators can refer at various points throughout the school year. Any necessary interventions are able to draw on a much broader scope of strengths, allowing more strength crossover between contexts, a continuation of the positive relationship with students and caregivers, and a concrete idea of students' goals and values for all of the people involved in the process. For example, if a child is acting out behaviourally with peers at school, yet has the strengths of being responsible and being a role model for siblings at home, it can be useful for staff in the school to explore student's strengths at home with the student and a caregiver, and to identify what changes can be made for the student to carry over his or her strengths from the home context to the school. Carrying over strengths from one domain to another is made possible by also assessing strengths that occur outside of the school context.

A simple and comprehensive method of assessing students' strengths is by administering a questionnaire that uses multiple sources of information as well as assessing various contexts and developmental aspects of students (Rawana \& 
Brownlee, 2009). The Strengths Assessment Inventory (S.A.I.) (Rawana \& Brownlee, 2010) is a questionnaire developed specifically for the purpose of assessing a broad spectrum of students' individual strengths. The S.A.I. assesses strengths from five naturally occurring domains of functioning (Peers, Family and Home, School, Employment, and Community) as well as five personal developmental domains (Personality, Personal and Physical Care, Leisure and Recreation, Spiritual and Cultural Development, and Current and Future Goals). These domains are assessed, using the S.A.I. questionnaire, by the student's selfreport as well as by any significant people in the student's life, such as, a parent or caregiver, family member, educator, educational assistant, social worker, elder, or spiritual leader.

However, when the option of assessment of strengths is not available, there are many viable alternatives, including discussions about strengths with students, caregivers, other educators and staff members, and in class, incorporated into class activities, by school counsellors or psychologists (Saleebey, 2008), or with resources already being accessed outside of the school, such as social workers. Ideally, more than one of these approaches will be utilized, to achieve the broadest and more comprehensive conceptualization of students' strengths. The importance of drawing others into the discussion about an individual's strength, rather than relying solely on self-report, is that often other people will recognize characteristics, capacities, and resources that individuals do not realize about themselves. It can also be useful to have these discussions at various points throughout the school year as development, changes, and new experiences can be noted and reflected upon as the strengths picture develops.

In the classroom, the exploration stage has the potential to be enhanced throughout the school year, reflecting the developing strengths of students, as well as their deeper understanding of and self-reflection on their individual strengths and the strengths of their peers. Cathy described the strengths wall that she implemented in her Grade 4 classroom and the benefit of continual reflection on the strengths of students. At the beginning of the year, when the class discussed strengths, the strengths the students self-identified were largely skills or talents, rather than internal strengths or character strengths. Students said that they were good hockey players or good artists, and these strengths were posted on the strengths wall. However, after that, the bulletin board "became wallpaper" with no clear purpose of meaning for the class. In an effort to revitalize it, Cathy began to explore the strengths of her students, with concrete examples, as they arose in the contexts of the school and classroom. For example, Cathy said an example of what she might say would be, "Wow, you know what I noticed class? I noticed that Henry is really giving. He doesn't mind sharing any of his belongings," and she would post that on the strengths wall, under Henry's name. This process continued until the students began to mirror this behaviour, pointing out their peer's strengths with concrete examples. Cathy created a spot on her desk where the students could write the strengths of anyone else on a sticky note for her to write up and attach to the Strengths Wall: "They write about almost anyone who comes into our class. They write it about the student teacher, they write it about me, all about each other. So I'll just put them on cards and we'll put them up. And they refer to them in class. If things happen on the playground, we'll refer to the strengths wall." The strengths wall has become a dynamic part 
of the classroom culture, promoting students' self-reflection, self-esteem, and self-image. This effective method of exploration is suitable for the classroom and is preparation for the next stage of the strengths intervention, expansion.

\section{Expansion}

After strengths have been identified, it is necessary for students to be aware of what they can use those strengths to achieve, accomplish, and overcome. This involves reflection on the part of the student as well as reflection by those significant people involved in the student's life (Rawana \& Brownlee, 2009). In the context of school, the shift of awareness of the student's strengths needs to happen not just on the part of the classroom teacher, but also on the part of peers, support staff, other educators with whom the student may interact, the principal, and, of course, the student's caregivers. Although this seems at first like an idealized and impractical situation when the strengths, assessment, and treatment model is undertaken by a school community, a shift into a culture of strengths becomes possible (Brownlee, Rawana, MacArthur, \& Probizanski, 2010). As a result, what is valued by the student becomes apparent not only to those working with the student, but also to the student himself or herself, and an identity is formed within the student that is reinforced by the environment to which the student is exposed daily. What becomes reinforced is an identity of the student as a person who has positive qualities as well as individual strengths and assets, rather than as a person who is 'the bad kid' or someone who lacks any positive qualities.

In a counselling situation, this is accomplished by ongoing conversation with the client to uncover exactly how the strengths can be applied to the challenges the client faces (Rawana \& Brownlee, 2009). In the classroom, this process is not practical to the same extent because the educator is generally working with a room full of students and may not have as many opportunities to do extensive one-on-one work. Nonetheless, there are a number of ways to work around this situation. The method of working strengths into the curriculum has been successful in respect to literacy (Cadell et al., 2005). Looking at the strengths of characters in the stories the class is reading and engaging the class in discussion about how the characters used these traits, Cathy noted she has been able to reflect with the class as a group on how people use their strengths to accomplish goals. By analyzing how characters are able to use their strengths or how the characters do not use their strengths, within the story, students can increase their appreciation of the ways in which people choose to use their strengths. When these connections can be made theoretically, both about characters in stories as well as in the students' lives, students can begin to consider how their own knowledge, capacities, and resources can be used to find solutions to problems in their lives, and to achieve their goals (Saleebey, 2006). In one activity, Cathy had the students list the strengths of the characters the class had read about during the year as well as how they used their strengths. Following this curriculum-based warm up, the class paired off and, after a brief discussion, listed a strength of their partner. Following this, they listed strengths of anyone in the class. The students were very conscious on ensuring that everyone had a strength listed and even included a student that had entered the room mid-activity with his educational assistant, and had to leave the room early for special programming. 
The students noticed on their own that the student who was late in joining the group had been left out of the strengths activity due to his altered schedule and ensured his strengths were written down. The strength they observed was that the student had a "photographic memory," turning one of the characteristics that made him different from many of the other students into his strength. The types of strengths the Grade 4 class was observing in each other were characteristics, such as, being intelligent, kind, funny, cheerful, a great friend, a go-getter, responsible, positive, a leader, and respectful. After the activity, a student pointed out that everyone had different strengths, but they were all equally important. Once the students recognized that all sorts of strengths could be of value they were seen as ready to implement the action phase of change, which is labelled the evolution stage.

\section{Evolution}

The evolution stage is the point in the strengths intervention process that active change is undertaken. Up to this phase, attitudes, perspectives, and opinions have been explored and revealed. Evolution occurs when an educator takes the strengths that have been assessed, and, using the positive foundation on which the relationship with the student is built, respectfully challenges the students to use their strengths in productive ways-academically, behaviourally, emotionally, and socially, maximizing their potential.

The strength intervention prompts students to see that the people around them value them and believe in their potential, even if the students cannot see this potential themselves. In his discussion about the use of the strengths perspective by school counsellors, Saleebey reminds us that "if just one person is steadfast in his or her belief in a child, it can make a significant difference in how that child feels about himself or herself" (2006, p. 69). Educators accomplish this stage of the intervention in simple ways within the school and classroom, and even within the curriculum. With the Strengths Wall as a concrete record of the strengths of the students, the educator can use the students' strengths as a springboard to discuss potential, goals, challenges, and problems. If the students have identified with the Strengths Wall, these strengths become an achievable goal that has been set, and to which the student can aspire. If a student is experiencing problems in one area, yet has strengths in another (as everyone does), the educator and student can discuss how to utilize the strengths to overcome the challenges, finding a solution that best suits the individual and the situation.

Reminding students of what their strengths are is another approach that has been successful. Simply by pointing out that students are not working with their strengths and pointing out the internal resources they have, educators can reroute conflict and frustration. If a teacher believes that there are underlying or unexpressed issues causing students to not be performing at their best, the previous discussions about character strengths can be drawn upon to help students solve problems, and regain confidence. In addition, due to the nature of a strengths-based relationship with their educators, students may be willing to have a discussion, rather than to "turn off" when being spoken to. The reverse of this is also true; educators who have recognized their students' strengths and respect and value what students offer are less likely to give orders and are more 
likely to discuss issues. In this dialogue, educators and students can together review students' strengths and discuss how to best use them in situations in which students may be struggling and to get ahead in areas students want to get ahead. This is the point in the strengths intervention in which the plan is made and evaluated in which the student's personal agency is combined with the available resources to implement change (Rawana \& Brownlee, 2009).

When schools and educators begin to tap the strengths of their students and to shift thinking from deficit to strength, students may begin to realize their potential within the school. The next section describes the experiences of a educator who implemented the strength, assessment, and treatment model within her classroom for one year, and demonstrates both the amazing potential that can be realized by students who learn that they have something to offer, as well as some of the limitations of the intervention. When the concept of strengths is harnessed and teamed with the resources that schools, families, communities, and individuals can offer, the result is often that students rise above their deficits, issues, and the labels that have been put on them, and that the resilience of everyone, and not only those who have already overcome the odds, is increased.

\section{Case Studies}

\section{Case One}

Jane, a ten-year-old pupil, began the school year with the awareness that she had an altered curriculum, and made a point of informing the educator that, "I don't do regular work. I do special work." In addition to working below her grade level, she was also struggling with reading, and was not invested in the classroom literacy initiatives. She was a "fake reader," in that she would pretend to read books very quickly and would be left with nothing to do during the literacy time in the classroom. When the rest of the class was completing their literacy, Jane began to create questions, modelled on what the class was reading, on her own initiative. She would reproduce them at home, using her own time and supplies, and began to ask the educator if her literacy group could complete them when they were not doing guided reading. Recognizing Jane's strengths as a leader and the initiative she had taken to create this work for her group in a contextual domain that would traditionally be considered a deficit area, the educator allowed Jane to explore this new role as a peer instructor. Jane was modelling the positive behaviours undertaken by the class. In addition, the whole class became interested in Jane's work, and wanted to be involved, which the educator allowed. The class became very self-directed at that time of day, due to Jane's leadership and initiative, and the educator was receiving excellent work from them all, as a result of their investment and self-direction.

Jane's strengths were being recognized by the educator and her classmates on the Strengths Wall. The exploration of her strengths as a leader, and the expansion of her strengths from supporting other to focusing on her own work resulted in Jane re-envisioning herself as a good student, investing in her learning, and leaving behind her identity as the kid who gets special work, resulting in her move to grade-level work. Jane is now often the first student to complete her work, she no longer has an altered curriculum, and she supports her peers with their schoolwork as well. She has also participated in the school's 
Reading Buddies program with the Junior Kindergarten class, and has invested herself in this program to the point that when she was too ill to attend school, her caregiver reported that she was upset because she did not want to let her reading buddy down.

Jane's educator reported that Jane understands that she is a leader, and that leadership behaviour is expected of her in the school. She has a positive vision of herself to which she now aspires, which is also recognized by her peers, educators, and caregivers. Her educator said, "I'm proud of her. And she's done it, she's owned it."

\section{Case Two}

John, a ten-year-old male pupil, was described by his educator as "a work in progress." John's academic and behavioural issues present major challenges to the staff, as well as to the classroom dynamics. John will submit work that is scribbled on and completely illegible, and is frequently unable to stay on track. The educator has noted self-esteem issues as well, relating to his behavioural issues in the classroom and the playground. John's strengths have been explored by the educator, and enhanced by his peers through the ongoing use of strengths within the classroom. When John is experiencing the academic or behavioural challenges with which he copes, his educator has found that the strengths intervention within the classroom setting is an effective method of getting the outcomes that she know he is capable of producing. Posted on the strengths wall in the classroom are the positive attributes that have been recognized in John by staff and peers. If John submits work that is not legible, she explores his strengths with him and has found that when she calls his attention to his creativity, and challenges him to use that creativity to produce quality work, work that she has seen him produce and of which she knows he is capable. The result is described as being as though a different student has handed it in. Similarly, when John has conflicts with other students, his educator is able to explore what is going on with him. She asks him about why he is acting out, and recognizes that "You are not that kid." His strengths, such as "being funny," and "being fun to play with," reinforce what his peers respect and value in him as an individual. Nonetheless, John has attempted to challenge the Strengths Wall, saying, " 'Being nice' isn't one of my strengths." However, as the strengths he has have been formally recognized by the people with whom he interacts daily, staff are able to expand upon them to highlight the fact that he is a good kid, and that people like him. His educator recognizes that the strengths intervention is a positive influence on John, and will support any further specific interventions that need to occur, complementing what needs to happen.

\section{Conclusion}

The strengths, assessment, and treatment model implemented by the school in this paper has positively impacted the entire school community. When schools are perceived as caring, pro-social, and inclusive environments, classrooms are more likely to be protective factors in students' lives (Cefai, 2007). The strengths perspective, when implemented by principals and educators, has the potential to increase this experience for students and their families. The intervention not only 
transforms the way the educators approach their profession and the ways in which they interact with students, but the intervention also changes the way that students perceive themselves, and the potential that they see in themselves. The strengths model recognizes strengths in everyone, which means that students are not singled out for needing extra support and students whose challenges might have escaped particular notice also receive a positive intervention. By working on transferring strengths between various contexts, the students learn life skills that can increase their resilience in many contexts throughout their lives. Working on transferring strengths also gives educators the opportunity to increase the protective factors in the lives of their students, as well as to help transform negative behaviours or detrimental situations with their students into something more constructive. The strengths perspective aligns educators with students and families, perceivably demonstrating that they can work together. As the teacher observed, important people in students' lives "know that you value their child." In addition, the student, through the process of strengths work, may feel much more motivated to address issues that need to be remedied. Thus, strengths become the assets used to address academic and behavioural difficulties.

\section{Acknowledgement}

The authors would like to thank the Ontario Education Services Corporation for providing funding towards this research and Michelle Probizanski and Heather Harris for their help and assistance with this research.

\section{References}

Brown, J. H., D’Emidio-Caston, M., \& Benard, B. (2001). Resilience education. Thousand Oaks, CA: Corwin.

Brownlee, K., Rawana, E. P., MacArthur, J., \& Probizanski, M. (2010). The culture of strengths makes them feel valued and competent: Aboriginal children, child welfare, and a school strengths intervention. First Peoples Child and Family Review, 5, 96-103.

Cadell, S., Fletcher, M., Makkappallil-Knowles, E., Caldwell, S., Wong, L., Bodurtha, D., et al. (2005). The use of the arts and the strengths perspective: The example of a course assignment. Social Work Education: The International Journal, 24, 137-146.

Cefai, C. (2007). Resiliency for all: A study of classrooms as protective contexts. Emotional and Behavioural Difficulties, 12, 119-134.

Condly, S. J. (2006). Resilience in children: A review of literature with implications for education. Urban Education, 41, 211-236.

Cooper, C. E. \& Crosnoe, R. (2007). The engagement in schooling of economically disadvantaged parents and children. Youth Society, 38, 372-391.

Farmer, T. W., Farmer, E. M. Z., \& Brooks, D. S. (2010). Recasting the ecological and developmental roots of intervention for students with emotional and behavior problems: The promise of strength-based perspectives. Exceptionality, 18, 53-57.

Fredricks, J. A., Blumenfeld, P. C., \& Paris, A. H. (2004). School engagement: Potential of the concept, state of the evidence. Review of Educational Research, 74, 59-109.

Glicken, M. D. (2004). Using the strengths perspective in social work practice. New York, NY: Pearson.

Howard, S., Dryden, J., \& Johnson, B. (1999). Childhood resilience: Review and critique of literature. Oxford Review of Education, 25, 307-323.

Jimerson, S. R. (2004). The California School Psychologist provides valuable information 
regarding strength-based assessment, youth development, and school success. The California School Psychologist, 9, 3-7.

Luthar, S. S., Cicchetti, D., \& Becker, B. (2000). The construct of resilience: A critical evaluation and guidelines for future work. Child Development, 71, 543-562.

Masten, A. S. (2001). Ordinary magic: Resilience processes in development. American Psychologist, 56, 227-238.

Masten, A., Herbers, J., Cutuli, J., \& Lafavor, T. (2008). Promoting Competence and Resilience in the School Context. Professional School Counseling, 12, 76-84.

Osterman, K. F. (2000). Students' need for belonging in the school community. Review of Educational Research, 70, 323-367.

Rawana, E. P. \& Brownlee, K. (2009). Making the possible probable: A strength-based assessment and intervention framework for clinical work with parents, children and adolescents. Families in Society: The Journal of Contemporary Social Services, 90, 255-260.

Rawana, E. P. \& Brownlee, K. (2010). The Strength Assessment Inventory for Children and Adolescents (Revised). Thunder Bay, ON: Centre of Excellence for Children and Adolescents with Special Needs.

Rawana, E. P., Brownlee, K., Whitley, J., Rawana, J., Franks, J., \& Walker, D. (2009). Strengths in Motion: A strengths-based approach to enhance positive school climate and address issues of bullying and school violence. Thunder Bay, ON: Centre of Excellence for Children and Adolescents with Special Needs.

Saleebey, D. (Ed.). (1997). The strengths perspective in social work practice (2nd ed.). Toronto, Canada: Pearson Education.

Saleebey, D. (2006). Power in the people. In Saleebey, D. (Ed.), The strengths perspective in social work practice (4th ed.) (pp. 1-23). Toronto, Canada: Pearson Education.

Saleebey, D. (2008). Commentary on the strengths perspective and potential applications in school counseling. Professional School Counseling, 12, 68-75.

Solomon, D., Watson, M., Battistich, V., Schaps, E., \& Delucchi, K. (1996). Creating classrooms that students experience as communities. American Journal of Community Psychology, 24, 719-748.

Stewart, D., Sun, J., Patterson, C., Lemerle, K., \& Hardie, M. (2004). Promoting and building resilience in primary school communities: Evidence from a comprehensive "health promoting school" approach. International Journal of Mental Health Promotion, 6(3), 26-33.

Tedeschi, R. G., \& Kilmer, R. P. (2005). Assessing strengths, resilience and growth to guide clinical interventions. Professional Psychology: Research and Practice, 36, 230-237.

Ungar, M., Brown, M., Liebenberg, L., Cheung, M. \& Levine, K. (2008). Distinguishing differences in pathways to resilience among Canadian youth. Canadian Journal of Community Mental Health, 27, 1-13.

Werner, E. E., \& Smith, R. S. (1992). Overcoming the odds: High risk children from birth to adulthood. Ithaca, NY: Cornell University Press.

Keith Brownlee is the Director of Research for the Centre of Excellence for Children and Adolescents with Special Needs and Professor, School of Social Work, Lakehead University, Thunder Bay, Ontario. Email: kbrownle@lakeheadu.ca

Edward P. Rawana is the Director of the Centre of Excellence for Children and Adolescents with Special Needs and Associate Professor, Department of Psychology, Lakehead University, Thunder Bay, Ontario. Email: erawana@lakeheadu.ca

Julia MacArthur is an MSW Graduate of the School of Social Work, Lakehead University, Thunder Bay, Ontario. 